\title{
Uso de microminerais sob a forma de complexo orgânico em rações para frangas de reposição no período de 7 a 12 semanas de idade ${ }^{1}$
}

\author{
Jerônimo Ávito Gonçalves de Brito ${ }^{2}$, Antônio Gilberto Bertechini ${ }^{3}$, Édison José Fassani ${ }^{4}$, \\ Paulo Borges Rodrigues ${ }^{3}$, Rilke Tadeu Fonseca de Freitas ${ }^{3}$
}

\footnotetext{
${ }^{1}$ Parte dos resultados da dissertação do primeiro autor.

2 Doutorando em Zootecnia da UFLA.

${ }^{3}$ Departamento de Zootecnia - UFLA.

${ }^{4}$ Departamento de Zootecnia - UNIFENAS.
}

RESUMO - O experimento foi realizado objetivando-se estudar a utilização dos microminerais na forma de complexo orgânico em rações para frangas no período de 7 a 12 semanas de idade e sua influência no desempenho e nas características ósseas. Os tratamentos consistiram da suplementação dos microminerais nas formas inorgânica (tratamento controle) e orgânica com inclusão de $0,4 \%$ na ração, que corresponde aos níveis suplementares de 60, 80, 70, 10, 1 e 0,3 ppm de Zn, Fe, $\mathrm{Mg}, \mathrm{Cu}, \mathrm{I}$ e Se, respectivamente, e na redução dos níveis de inclusão da fonte orgânica nas rações $(0,35 ; 0,3 ; 0,25$ e $0,2 \%)$ Seiscentos e quarenta e oito frangas Lohmann - LSL, iniciando a 7 a semana de idade, foram utilizadas em um delineamento inteiramente casualizado, com seis tratamentos, seis repetições e 18 aves por parcela. Não houve diferenças significativas entre os tratamentos para características de desempenho e uniformidade até a $12^{\underline{a}}$ semana. Com a redução nos níveis de inclusão do suplemento orgânico, houve redução linear do teor de cinzas na tíbia das frangas na 12 a semana, porém, não houve diferenças entre a fonte inorgânica (tratamento controle) e os demais tratamentos (fonte orgânica). O teor de $\mathrm{Zn}$ em tíbias, assim como o peso, o comprimento, a espessura da tíbia e do fêmur e o comprimento do metatarso, não foi influenciado pelos tratamentos na 12 a semana. A fonte orgânica dos microminerais apresentou vantagem comparativa, possibilitando a redução no nível de inclusão da fonte orgânica para $0,2 \%$ na ração, sem afetar negativamente as características estudadas no período de 7 a 12 semanas de idade das frangas.

Palavras-chave: características ósseas, desempenho, frangas, suplemento mineral

\section{Effects of feeding trace minerals as organic complex for replacement pullets in the period from 7 to 12 weeks old}

\begin{abstract}
This experiment was carried out to study the effects of feeding trace minerals as organic complex on the performance and bone characteristics for pullets in the period from 7-12 weeks old. The treatments consisted of the supplementation of trace minerals in the inorganic form (control) and organic form with inclusion of $0.4 \%$ in the diet, that corresponds to the supplement levels of $60,80,70,10,1$, and $0.3 \mathrm{ppm}$ of zinc, iron, manganese, copper, iodine and selenium, respectively, and the decreasing dietary levels of organic source $(0.35,0.3,0.25$, and $0.2 \%)$. A total of 648 Lohmann - LSL pullets from the $7^{\text {th }}$ week old were allotted to a completely randomized design with six treatments and six replicates of 18 birds. No significant treatment effect on performance characteristics and uniformity up to the $12^{\text {th }}$ week was observed. There was a linear reduction of ash porcentage in the tibia of pullets in the $12^{\text {th }}$ week as the dietary organic supplement decreased However there were no significant differences among the inorganic source (control) and the other treatments (organic source) The zinc content in the tibia as well as the weight, length and thickness of tibia and femur and metatarsus length were not affected by the treatments, in the $12^{\text {th }}$ week. The trace mineral organic source showed relative comparative advantage and resulted in $0.2 \%$ reduction of the dietary supplement level of organic source with no effect on the performance and bone characteristics of pullets from 7 to 12 weeks old.
\end{abstract}

Key Words: bone characteristics, mineral supplement, performance, pullets

\section{Introdução}

A evolução do desempenho de poedeiras comerciais foi bastante substancial nos últimos anos. Atualmente, são encontradas linhagens cada vez mais precoces e produtivas, sendo consenso geral que fases de cria e recria estão diretamente relacionadas ao sucesso na fase de produção.

Os microminerais são considerados de grande importância na alimentação das aves, pois participam de uma série de processos bioquímicos, essenciais ao crescimento e 
desenvolvimento, destacando a formação óssea. A maioria dos microminerais (zinco, cobre, manganês, iodo e ferro) está associada direta ou indiretamente, sendo a fase de 7 a 12 semanas em frangas de reposição responsável pelo maior crescimento proporcional do esqueleto.

Tendo-se em vista as perspectivas de maior biodisponibilidade em relação a fontes inorgânicas convencionais, os microminerais sob a forma de complexo orgânico têm sido pesquisados. Partindo do pressuposto de que são mais facilmente absorvidos e retidos pelas aves, fontes orgânicas de suplementação de minerais podem atuar melhorando o desempenho e a vida útil das aves e reduzindo a excreção dos microminerais que potencialmente poluem o ambiente. Todavia, os resultados ainda são contraditórios.

Os microminerais $\mathrm{Zn}, \mathrm{Mg}$ e Cu estão diretamente associados ao crescimento e ao desenvolvimento do tecido ósseo (Underwood, 1999). O zinco é um dos constituintes da metaloenzima anidrase carbônica $(0,3 \%)$ e atua no equilíbrio ácido-base, no organismo e na calcificação óssea (Leeson \& Summers, 2001). Os tecidos muscular e ósseo são os principais tecidos de reserva de zinco e possuem capacidade de liberar possíveis excedentes em condições de deficiência na dieta (Emmert \& Baker, 1995; Underwood, 1999). O manganês é ativador metálico das enzimas envolvidas na síntese de mucopolissacarídeos e glicoproteínas que contribuem na formação da matriz orgânica dos ossos e da casca dos ovos (Georgievski, 1982). Segundo Scott et al. (1982), o cobre desempenha papel importante na formação óssea, sendo ativador da lisil oxidase, enzima que participa da biossíntese de colágeno.

Os microminerais Fe, I e Se participam de reações de síntese de inúmeros compostos importantes no organismo da ave em crescimento, como a hemoglobina e os hormônios tireoideanos, e na manutenção da integridade das membranas biológicas (Mcdowell, 1992).

A AAFCO (1997) conceitua os minerais orgânicos como íons metálicos ligados quimicamente a uma molécula orgânica, formando estruturas com características únicas de estabilidade e de alta biodisponibilidade mineral.

Segundo Baruselli (2003), novas técnicas de quelação permitiram desenvolver novos produtos como os carboquelatos, que consistem na lise enzimática de leveduras específicas, fermentados sobre um substrato aditivado com fósforo (fosforilação) e íons metálicos formando complexos orgânicos muito ricos em metabólitos e de alta biodisponibilidade.

Pimentel et al. (1991) não verificaram diferenças na biodisponibilidade relativa entre as fontes óxido de zinco e zinco-metionina para frangos. Baker et al. (1991) constata- ram que o cobre do complexo cobre-lisina e o cobre na forma de sulfato de cobre foram semelhantes quanto ao aproveitamento por frangos de corte, ao passo que o óxido de cobre apresentou menor disponibilidade.

Aoyagi \& Baker (1993a), avaliando fontes de cobre do complexo cobre-metionina e do sulfato de cobre, estimaram valores de 96 e $88 \%$ de biodisponibilidade utilizando as concentrações de cobre na bile e no fígado de frangos, respectivamente.

Aoyagi \& Baker (1993b) verificaram que o valor biológico do zinco do quelato zinco-lisina foi $111 \%$, em comparação ao sulfato de zinco (100\%), utilizando-se a concentração de zinco nos ossos como característica avaliada.

A disponibilidade do manganês proveniente de diferentes fontes de suplementação foi determinada por Smith et al. (1995), em experimento utilizando frangos de corte. Os valores de biodisponibilidade das fontes óxido de manganês e proteinato de manganês em relação ao sulfato de manganês (100\%) foram de 91 e $120 \%$ (21 dias) e de 83 e $125 \%$ (49 dias), respectivamente.

Cao et al. (1996) verificaram, em ensaio de disponibilidade biológica com fontes de ferro para frangos de corte $\left(\mathrm{FeSO}_{4}\right.$ reagent-grade, $\mathrm{FeSO}_{4}$ feed-grade e ferrometionina) que a forma orgânica apresentou $88 \%$ de biodisponibilidade quando comparada à fonte reagentgrade, considerada padrão.

Entre os microminerais, o selênio sob a forma orgânica é o mais consolidado e estudado; existem evidências efetivas que, sob essa forma, este mineral é mais biodisponível (Arruda et al., 2004).

Um estudo conduzido por Leeson (2003) revelou que a substituição da fonte de suplementação (inorgânica por proteinato de microminerais) e a redução gradativa nos níveis suplementares dos microminerais $(\mathrm{Zn}, \mathrm{Mg}, \mathrm{Fe}$ e $\mathrm{Cu})$ não afetaram o desempenho de frangos de corte no período de 1 a 42 dias de idade. Entretanto, houve redução significativa na excreção de $\mathrm{Zn}, \mathrm{Cu}$ e $\mathrm{Mg}$ com a redução da suplementação dos microminerais pela forma orgânica.

Xavier et al. (2004) observaram melhorias nos índices de desempenho e qualidade dos ovos de poedeiras semipesadas no segundo ciclo de produção e concluíram que existem benefícios na inclusão de $\mathrm{Se}, \mathrm{Zn}$ e $\mathrm{Mg}$ sob a forma de complexo orgânico nesta fase.

O NRC (1994) preconiza níveis de suplementação para frangas de reposição de 30;35; 60; 4; 0,35 e 0,10 (ppm) de $\mathrm{Mg}, \mathrm{Zn}, \mathrm{Fe}, \mathrm{Cu}, \mathrm{I}$ e Se, respectivamente, para a fase de 6 a 18 semanas de idade, enquanto Rostagno et al. (2000) recomendam, para as condições brasileiras, níveis de suplementação ppm desses minerais de $70 ; 60 ; 50 ; 8,5 ; 1 \mathrm{e}$ 0,25 respectivamente, para todas as categorias de explora- 
ção avícola industrial (frangos de corte, aves de reposição, postura e matrizes).

Objetivou-se com este trabalho estudar a utilização dos principais microminerais essenciais na forma de complexo orgânico em rações para frangas de reposição no período de 7 a 12 semanas e sua influência no desempenho e nas características ósseas.

\section{Material e Métodos}

O experimento foi realizado no Setor de Avicultura do Departamento de Zootecnia da Universidade Federal de Lavras. Foram utilizadas 648 frangas da linhagem LohmannLSL com 42 dias de idade, alojadas em galpão convencional de recria com cobertura de telhas de amianto, distribuídas em quatro fileiras de gaiolas com divisões de $50 \times 45 \times 38 \mathrm{~cm}$, contendo comedouros e bebedouros tipo calha, com escoamento contínuo de água. As aves foram vacinadas contra os principais desafios da região e, na $10 \underline{\underline{a}}$ semana de idade, foram submetidas à segunda debicagem.

Utilizou-se o delineamento inteiramente casualizado, com seis tratamentos e seis repetições. A parcela experimental foi constituída de 18 aves e os tratamentos experimentais consistiram da inclusão de $0,4 \%$ de um suplemento inorgânico de microminerais na ração (tratamento controle) e cinco níveis de inclusão de um suplemento de microminerais na forma de complexo orgânico $(0,4 ; 0,35 ; 0,30$; $0,25$ e $0,20 \%)$. A redução gradativa $(0,05 \%)$ nos níveis do suplemento de microminerais sob a forma orgânica na ração foi feita simultaneamente com a inclusão de caulim nas rações.

Os níveis de cada micromineral, conforme os tratamentos, são apresentados na Tabela 1.

Os suplementos de microminerais (inorgânico e orgânico) foram elaborados por uma empresa comercial, para fins de pesquisa, com os níveis de garantia para atender às recomendações nutricionais de frangas de reposição e frangos de corte. Utilizaram-se leveduras e soluções (sais de cada micromineral em estudo) para incorporação dos microminerais em quantidades específicas e obtenção da concentração desejada. A análise referente à concentração dos microminerais (exceto Se e I) nas fontes utilizadas foi realizada segundo AOAC (1990) e os resultados são apresentados na Tabela 2.

A rações experimentais (Tabela 3), à base de milho e farelo de soja, foram formuladas de acordo com o programa alimentar recomendado para a linhagem, sendo isonutrientes, com exceção dos níveis de microminerais, que constituíram os tratamentos. A composição dos principais alimentos utilizados na formulação foi obtida nas tabelas brasileiras (Rostagno et al., 2000).

No final da $12 \underline{\underline{a}}$ semana, as aves, assim como as sobras das rações experimentais, foram pesadas individualmente. Duas aves por parcela (12 por tratamento) foram abatidas para retirada da tíbia e do fêmur para análises posteriores. As características avaliadas no período de 7 a 12 semanas de idade foram o ganho de peso (g/ave), a conversão alimentar (g ração/g ganho de peso), o consumo de ração (g/ave) e a uniformidade ( $\pm 10 \%$ da média do peso corporal das aves de cada tratamento). Foram realizadas a medição do comprimento do metatarso ( $\mathrm{cm} / \mathrm{kg}$ peso vivo) e a determinação dos teores de cinzas (\%) e zinco $(\mathrm{mg} / \mathrm{kg})$ na tíbia das aves abatidas, segundo AOAC (1990).

A determinação do comprimento e da espessura dos ossos foi feita por meio de paquímetro e o peso dos ossos foi obtido com auxílio de balança de precisão $(0,0001 \mathrm{~g})$, sendo os resultados expressos com base na matéria seca desengordurada.

Os dados foram submetidos à análise estatística utilizando-se o Sistema de Análise de Variância para dados balanceados (Sisvar), descrito por Ferreira (2000), procedendo-se às análises de regressão para os níveis do suplemento sob a forma de complexo orgânico. Foi realizado o teste Dunnet a 5\% de probabilidade para comparação de cada nível de suplementação do complexo orgânico com o tratamento controle (inorgânico).

Tabela 1 - Suplementação dos microminerais de acordo com as fontes e os níveis de inclusão estudados Table 1 - Trace minerals supplementation according to the sources and inclusion levels

\begin{tabular}{|c|c|c|c|c|c|c|}
\hline \multirow[t]{2}{*}{$\begin{array}{l}\text { Micromineral }(\mathrm{mg} / \mathrm{kg}) \\
\text { Trace mineral }(\mathrm{mg} / \mathrm{kg})\end{array}$} & \multirow{2}{*}{$\begin{array}{c}\text { Controle } \\
\text { Control } \\
0,40 \mathrm{SI}\end{array}$} & \multicolumn{5}{|c|}{$\begin{array}{l}\text { Nível suplementar }{ }^{1}(\%) \\
\text { Supplement level (\%) }\end{array}$} \\
\hline & & $0,40 \mathrm{SO}$ & $0,35 \mathrm{SO}$ & $0,30 \mathrm{~S}$ & $0,25 \mathrm{SO}$ & $0,20 \mathrm{SO}$ \\
\hline $\mathrm{Fe}$ & 80 & 80 & 70 & 60 & 50 & 40 \\
\hline $\mathrm{Mg}$ & 70 & 70 & 61,25 & 52.2 & 43,75 & 35 \\
\hline $\mathrm{Cu}$ & 10 & 10 & 8,75 & 7,5 & 6,25 & 5 \\
\hline
\end{tabular}

1 SI: suplemento inorgânico; SO: suplemento orgânico.

1 SI: inorganic source; SO: organic source. 
Tabela 2 - Análise do teor dos microminerais nos suplementos ${ }^{1}$ Table 2 - Analysis of the trace mineral content in the supplements

Micromineral Comp. orgânico $(\mathrm{mg} / \mathrm{kg})$ Inorgânico $(\mathrm{mg} / \mathrm{kg})$ Trace mineral Organic complex $(\mathrm{mg} / \mathrm{kg}) \quad$ Inorganic $(\mathrm{mg} / \mathrm{kg})$

\begin{tabular}{lrr}
\hline $\mathrm{Zn}$ & 15.570 & 14.850 \\
$\mathrm{Fe}$ & 16.000 & 17.000 \\
$\mathrm{Mg}$ & 21.750 & 22.020 \\
$\mathrm{Cu}$ & 2.560 & 2.260 \\
\hline
\end{tabular}

${ }_{1}^{1}$ Análises realizadas no Laboratório de Pesquisa Animal/DZO/UFLA.

${ }^{1}$ Analysis were performed at the Animal Research Laboratory/DZO/UFLA.

\section{Resultados e Discussão}

Os resultados referentes ao desempenho das frangas de reposição submetidas às duas fontes e aos diferentes níveis dos microminerais na fase de 7 a 12 semanas são apresentados na Tabela 4.

As fontes e os níveis dos microminerais não influenciaram $(\mathrm{P}>0,05)$ o desempenho de frangas de reposição no

Tabela 3 - Composição percentual e níveis nutricionais calculados das rações experimentais

Table 3 - Ingredient composition and calculated nutritional levels of the experimental diets

\begin{tabular}{|c|c|c|c|c|c|c|}
\hline \multirow[t]{2}{*}{$\begin{array}{l}\text { Ingrediente (\%) } \\
\text { Ingredient (\%) }\end{array}$} & \multirow{2}{*}{$\begin{array}{c}\text { Controle } \\
\text { Control } \\
0,40 \text { SI }\end{array}$} & \multicolumn{5}{|c|}{$\begin{array}{l}\text { Nível suplementar* (\%) } \\
\text { Supplement level (\%) }\end{array}$} \\
\hline & & $0,40 \mathrm{SO}$ & $0,35 \mathrm{SO}$ & $0,30 \mathrm{~S}$ & $0,25 \mathrm{SO}$ & $0,20 \mathrm{SO}$ \\
\hline Farelo de soja (Soybean meal) & 28,320 & 28,320 & 28,320 & 28,320 & 28,320 & 28,320 \\
\hline Farelo de trigo (Wheat meal) & 4,714 & 4,714 & 4,714 & 4,714 & 4,714 & 4,714 \\
\hline Fostato bicálcico (Dicalcium phosphate) & 1,532 & 1,532 & 1,532 & 1,532 & 1,532 & 1,532 \\
\hline Sal comum (Salt) & 0,394 & 0,394 & 0,394 & 0,394 & 0,394 & 0,394 \\
\hline DL-metionina (DL-methionine) & 0,052 & 0,052 & 0,052 & 0,052 & 0,052 & 0,052 \\
\hline L-lisina (L-Lysine) & 0,038 & 0,038 & 0,038 & 0,038 & 0,038 & 0,038 \\
\hline Cloreto de colina (Choline chloride) & 0,035 & 0,035 & 0,035 & 0,035 & 0,035 & 0,035 \\
\hline Bacitracina de zinco (Zinc bacitracin) & 0,025 & 0,025 & 0,025 & 0,025 & 0,025 & 0,025 \\
\hline Suplemento vitamínico ${ }^{1}$ (Vitamin premix) & 0,100 & 0,100 & 0,100 & 0,100 & 0,100 & 0,100 \\
\hline $\mathrm{EM}(M E)(\mathrm{kcal} / \mathrm{kg})$ & 2.850 & 2.850 & 2.850 & 2.850 & 2.850 & 2.850 \\
\hline $\mathrm{PB}(C P)(\%)$ & 19,00 & 19,00 & 19,00 & 19,00 & 19,00 & 19,00 \\
\hline $\mathrm{Ca}(\%)$ & 1,10 & 1,10 & 1,10 & 1,10 & 1,10 & 1,10 \\
\hline Fósforo disponível (Available phosphorus) (\%) & 0,40 & 0,40 & 0,40 & 0,40 & 0,40 & 0,40 \\
\hline Metionina (Methionine) (\%) & 0,35 & 0,35 & 0,35 & 0,35 & 0,35 & 0,35 \\
\hline Lisina (Lysine) $(\%)$ & 1,00 & 1,00 & 1,00 & 1,00 & 1,00 & 1,00 \\
\hline $\mathrm{Met}+\mathrm{Cis}(\mathrm{Met}+\mathrm{Cys})(\%)$ & 0,663 & 0,663 & 0,663 & 0,663 & 0,663 & 0,663 \\
\hline Sódio (Sodium) (\%) & 0,19 & 0,19 & 0,19 & 0,19 & 0,19 & 0,19 \\
\hline
\end{tabular}

* SI: suplemento Inorgânico; SO: suplemento orgânico.

* SI: inorganic source; SO: organic source.

1 Enriquecimento por kg da dieta (amount/kg of diet): vit. A - 10.000 UI; vit. $\mathrm{D}_{3}-2.000 \mathrm{UI}$; vit. E - $20 \mathrm{UI}$; vit. $\mathrm{K}_{3}-2 \mathrm{mg}$; vit. $\mathrm{B}_{1}-2 \mathrm{mg}$; vit. $\mathrm{B}_{2}-4 \mathrm{mg} ;$ vit. $\mathrm{B}_{6}$ - $4 \mathrm{mg}$; vit. $\mathrm{B}_{12}$ - $20 \mathrm{mcg}$; ácido fólico (folic acid) - $1 \mathrm{mg}$; ácido pantotênico (pantothenic acid) - $10 \mathrm{mcg}$; niacina (niacin) - $30 \mathrm{mg}$; biotina (biotin) - $60 \mathrm{mg}$; vit. C $50 \mathrm{mg}$; antioxidante (antioxidant) $-125 \mathrm{mg}$.

2 Composição por kg do produto (composition/kg of product): Mn - $17.500 \mathrm{mg} ; \mathrm{Zn}-15.000 \mathrm{mg}$; Fe - $20.000 \mathrm{mg}$; Cu - $2.500 \mathrm{mg}$; I $250 \mathrm{mg}$; Se $75 \mathrm{mg}$.

período de 7 a 12 semanas e todas as características apresentaram comportamento condizente aos padrões do Manual da Linhagem Lohmann - LSL (2002) para a fase em estudo.

Estes resultados sugerem que os níveis suplementares de microminerais preconizados pelo NRC (1994), muito semelhantes ao utilizado neste experimento no menor nível de inclusão do complexo orgânico $(0,2 \%)$, podem ser adotados, pois não afetam o desempenho de frangas na fase estudada.

Os resultados obtidos diferem dos encontrados por Ferket et al. (1992), que observaram melhor na conversão alimentar e na viabilidade de perus ao reduzirem ( 75 e $67 \%$, respectivamente) a suplementação de $\mathrm{Zn}$ e $\mathrm{Mg}$ quelatados com metionina, em relação à fonte inorgânica destes minerais. Corroboram, no entanto, os encontrados por Leeson (2003), que não verificou diferenças no desempenho de frangos de corte aos 42 dias quando reduziu a suplementação dos principais microminerais em até $20 \%$ da fonte inorgânica, considerada controle.

Considerando o desempenho para avaliar a boa formação e o desenvolvimento corporal de frangas de reposição na fase de 7 a 12 semanas, a redução nos níveis de microminerais sob a forma de complexo orgânico mostrou-se eficiente. 
Possíveis causas seriam as menores necessidades nutricionais dos microminerais na fase estudada, o melhor aproveitamento (maior taxa de absorção e/ou menor quantidade de interações entre os minerais) destes minerais quando suplementados em níveis mais baixos, ou mesmo esses dois fatores associados.

Os resultados referentes às características ósseas das frangas submetidas às diferentes fontes e aos níveis dos microminerais na fase de 7 a 12 semanas são descritos na Tabela 5 .

Os resultados obtidos para porcentagem de cinzas nas tíbias das aves na $12^{\underline{a}}$ semana de idade submetidas ao tratamento controle $(0,4 \%$ do suplemento inorgânico) não se mostraram superiores $(\mathrm{P}>0,05)$ a nenhum dos níveis de suplementação dos microminerais sob a forma orgânica.

Houve diferença significativa $(\mathrm{P}<0,05)$ entre os níveis da fonte orgânica de microminerais sobre o teor de cinzas na 12 a semana, com efeito linear, ou seja, o teor de cinzas na tíbia diminuiu com a redução nos níveis de suplementação. $\mathrm{O}$ efeito linear revelou que a redução de $0,05 \%$ na suplementação da fonte orgânica (que corresponde à reduções de 7,$5 ; 10 ; 8,75 ; 1,25 ; 0,125$ e 0,0375 ppm suplementar de $\mathrm{Zn}, \mathrm{Fe}, \mathrm{Mg}, \mathrm{Cu}, \mathrm{Ie} \mathrm{Se}$, respectivamente) provoca redução de $0,25 \%$ no teor de cinzas em tíbias das aves na idade avaliada.

A fase de 7 a 12 semanas, por ser de maior crescimento e formação do tecido ósseo, pode ter respondido mais facilmente à suplementação dos microminerais sob a forma de complexo orgânico. Todavia, o organismo geralmente é capaz de regular eficientemente as variações em médio prazo, de modo que a fonte pode ter colaborado para que níveis mais baixos de suplementação promovessem o mesmo comportamento (em relação ao teor de cinzas) observado com níveis mais altos.
Ressalta-se que, apesar do efeito linear dentro dos níveis de microminerais sob a forma de complexo orgânico para cinzas na 12 a semana, não houve diferenças $(\mathrm{P}>0,05)$ entre o tratamento controle $(0,4 \%$ de suplementação inorgânica) e os demais, ou seja, mesmo o menor nível de suplementação orgânica $(0,2 \%)$ é estatisticamente igual ao tratamento controle.

O teor de zinco nas tíbias não foi influenciado $(\mathrm{P}>0,05)$ pelos tratamentos na idade avaliada. Os ossos, por serem local de reserva deste micromineral no corpo, é um importante indicador de possíveis deficiências ou problemas futuros na postura. Os níveis de $\mathrm{Zn}$ no ovo (casca e gema) são altos e dietas deficientes neste mineral ou a baixa biodisponibilidade podem ocasionar reabsorção óssea.

A análise estatística dos dados não revelou diferenças significativas $(\mathrm{P}>0,05)$ entre os tratamentos para comprimento, peso e espessura da tíbia e do fêmur. Os resultados das características estruturais dos ossos longos corroboram os encontrados por Ferket et al. (1992), que não verificaram diferenças significativas quando reduziram a suplementação de 80 e 120 ppm de manganês, respectivamente, para 20 e 40 ppm de zinco-metionina e manganêsmetionina, sobre problemas e anormalidades de pernas de perus. Por outro lado, esses autores verificaram melhores respostas para a fonte orgânica destes minerais quando compararam as duas fontes (sais de zinco e manganês com os quelatos) no mesmo nível de suplementação ( 80 e $120 \mathrm{ppm})$

É importante salientar que a formação e o desenvolvimento dos ossos longos são importantes para o desempenho de uma poedeira moderna, visto que problemas ósseos na fase de produção são responsáveis por grandes perdas, ocasionadas por altos índices de mortalidade, e problemas como má qualidade de casca e, conseqüentemente, dos

Tabela 4 - Médias de ganho de peso (GP), consumo de ração (CR), conversão alimentar (CA) e uniformidade (U) no período de 7 a 12 semanas de idade, segundo os níveis e as fontes de suplementação dos microminerais

Table 4 - Means of weight gain (WG), feed intake (FI), feed:gain ratio (F/G) and uniformity (U) in the period from 7 to 12 weeks old according to trace mineral supplement levels and sources

\begin{tabular}{|c|c|c|c|c|c|c|c|}
\hline \multirow[t]{2}{*}{$\begin{array}{l}\text { Característica }{ }^{1,2} \\
\text { Characteristic }\end{array}$} & \multirow{2}{*}{$\begin{array}{c}\text { Controle } \\
\text { Control } \\
0,40 \mathrm{SI}\end{array}$} & \multicolumn{5}{|c|}{$\begin{array}{l}\text { Nível suplementar* (\%) } \\
\text { Supplement level (\%) }\end{array}$} & \multirow[t]{2}{*}{ CV $(\%)$} \\
\hline & & $0,40 \mathrm{SO}$ & $0,35 \mathrm{SO}$ & $0,30 \mathrm{~S}$ & $0,25 \mathrm{SO}$ & $0,20 \mathrm{SO}$ & \\
\hline GP (g/ave) (WG, g/bird) & 536,9 & 531,9 & 536,9 & 524,6 & 528,3 & 530,0 & 2,69 \\
\hline CR (g/ave) (FI, g/bird) & 2.326 & 2.297 & 2.330 & 2.296 & 2.306 & 2.298 & 1,60 \\
\hline $\mathrm{CA}(\mathrm{g} / \mathrm{g})(F C, g / g)$ & 4,33 & 4,32 & 4,34 & 4,38 & 4,37 & 4,34 & 1,97 \\
\hline U (\%) & 99,20 & 95,37 & 96,19 & 95,37 & 98,15 & 95,37 & 4,39 \\
\hline
\end{tabular}

1 Teste Dunnet $(P>0,05)$.

1 Dunnet test $(P>0.05)$

2 Regression $(P>0.05)$ 
Tabela 5 - Teores de cinzas (\%) e zinco ( $\mathrm{mg} / \mathrm{kg}$ ) na tíbia, peso, comprimento e espessura da tíbia e do fêmur e comprimento do metatarso em relação ao peso vivo $(\mathrm{cm} / \mathrm{kg})$ das aves com 12 semanas de idade (na matéria seca desengordurada), segundo os níveis e as fontes de suplementação dos microminerais

Table 5 - Contents of ash (\%) and zinc ( $\mathrm{mg} / \mathrm{kg})$ in the tibia, weight, length, thickness of tibia and femur and metatarsus length in relation to the body weight $(\mathrm{cm} / \mathrm{kg})$ of pullets at $12^{\text {th }}$ week old (on free fat dry matter) according to the trace mineral supplement levels and sources

\begin{tabular}{|c|c|c|c|c|c|c|c|}
\hline \multirow[t]{2}{*}{$\begin{array}{l}\text { Característica }{ }^{1} \\
\text { Characteristic }\end{array}$} & \multirow{2}{*}{$\begin{array}{c}\text { Controle } \\
\text { Control } \\
0,40 \text { SI }\end{array}$} & \multicolumn{5}{|c|}{$\begin{array}{l}\text { Nível suplementar }{ }^{1}(\%) \\
\text { Supplement level }(\%)\end{array}$} & \multirow[t]{2}{*}{ CV $(\%)$} \\
\hline & & $0,40 \mathrm{SO}$ & $0,35 \mathrm{SO}$ & $0,30 \mathrm{~S}$ & $0,25 \mathrm{SO}$ & $0,20 \mathrm{SO}$ & \\
\hline Cinzas $(\%)^{2}(A s h, \%)$ & 60,30 & 61,09 & 60,71 & 60,55 & 60,47 & 59,96 & 1,16 \\
\hline $\mathrm{Zn}(\mathrm{mg} / \mathrm{kg})$ & 234,6 & 222,9 & 219,2 & 225,4 & 242,6 & 226,3 & 8,23 \\
\hline Peso tíbia (mg) (Tibia weight, $m g$ ) & 2.778 & 2.764 & 2.677 & 2.724 & 2.731 & 2.669 & 4,95 \\
\hline Comp.tíbia (mm) (Tibia length, $\mathrm{mm}$ ) & 103,4 & 103,5 & 102,1 & 103,4 & 103,3 & 101,9 & 1,64 \\
\hline Espes. tíbia (mm) (Tibia thickness, $\mathrm{mm}$ ) & 5,08 & 5,15 & 5,01 & 4,99 & 5,16 & 5,03 & 3,75 \\
\hline Peso fêmur (mg) (Femur weight, mg) & 1.979 & 1.971 & 1.932 & 1.956 & 1.888 & 1.918 & 4,50 \\
\hline Comprimento fêmur (mm) (Femur length, $\mathrm{mm}$ ) & 68,90 & 69,25 & 68,48 & 69,09 & 68,21 & 68,76 & 1,44 \\
\hline Espesussurafêmur (mm) (Femur thickness, mm) & 6,19 & 6,23 & 6,05 & 6,25 & 6,10 & 6,11 & 2,97 \\
\hline Metatarso $(\mathrm{cm} / \mathrm{kg})$ (Metatarsus, $\mathrm{cm} / \mathrm{kg}$ ) & 10,03 & 9,97 & 9,90 & 10,02 & 9,94 & 9,99 & 1,84 \\
\hline
\end{tabular}

1 Teste Dunnet (Dunnet test) $(\mathrm{P}>0,05)$.

${ }^{1}$ Regressão para níveis da fonte orgânica (Regression for levels organic source) $(P>0,05)$.

2 Efeito linear (linear effect) $(P<0,05) \hat{Y}=59,056+4,998 x\left(R^{2}=93,14 \%\right)$.

ovos. A carência de estudos sobre o uso de microminerais nas fases de cria e recria de frangas associando-os à formação óssea não permite tecer maiores comparações, sendo necessárias mais pesquisas nessa área.

\section{Conclusões}

A redução nos níveis de suplementação da fonte orgânica, nas condições em que foi realizado o experimento, conferiu-lhe vantagem comparativa em detrimento à fonte inorgânica, pois não afetou o desempenho e as características ósseas das aves, podendo, portanto, ser utilizada como fonte de suplementação para frangas de reposição.

O menor nível de suplementação da fonte orgânica $(0,2 \%)$, que corresponde à suplementação de $30 ; 40 ; 35 ; 5$; 0,5 e 0,15 ppm de $\mathrm{Zn}, \mathrm{Fe}, \mathrm{Mg}, \mathrm{Cu}, \mathrm{I}$ e Se, respectivamente, pode ser adotado, pois se mostrou igual ao tratamento controle para as características ósseas e manteve o desempenho das aves na fase estudada.

A complexação de microminerais com leveduras e sua utilização como fonte suplementar para frangas de reposição podem ser adotadas, pois esta fonte é tecnicamente eficiente em manter os desenvolvimentos ósseo e corporal adequados.

\section{Literatura Citada}

AMERICAN ASSOCIATION FEED CONTROL OFFICIALS AAFCO. Atlanta: 1997. 266p.

ASSOCIATION OF OFFICIAL AGRICULTURAL CHEMISTS AOAC. Official methods of analysis. 15.ed. Arlington: Association of Official Analytical Chemists, 1990. n.1. 1117p.
AOYAGI, S.; BAKER, D.H. Nutritional evaluation of a coopermethionine complex for chicks. Poultry Science, v.72, n.12, p.2309-2315, 1993a

AOYAGI, S.; BAKER, D.H. Nutritional evaluation of a cooperlysine and zinc-lysine complexes for chicks. Poultry Science, v.72, n.1, p.16-171, 1993b

ARRUDA, J.S.; RUTZ, F.; PAN, E. A. Influence os replacing dietary inorganic witch organic selenium on peformance of broilers In: ANNUAL SYMPOSIUM ON BIOTECHNOLOGY IN THE FEED INDUSTRY, 20., 2004, Lexington. Proceedings... Lexington: 2004. p.13.

BAKER, D.H.; ODLE, J.; FUNK, M.A. et al. Bioavailability of copper in cupric oxide, cuprous oxide, and in a copper-lysine complex. Poultry Science, v.70, n.1, p.177-179, 1991.

BARUSELLI, M.S. Efeito do uso de minerais orgânicos no desempenho e comportamento animal. In: CONGRESSO INTERNACIONAL DE ZOOTECNIA - ZOOTEC, 2003 , Uberaba. Anais... Uberaba: 2003 (CD-ROM)

CAO, J.; HENRY, P.R.; AMMERMANN, C.B. et al. Effect of dietary iron concentration, age and length of iron for use on feed intake and tissue iron concentration of broiler chicks for use as a bioassay supplemental iron sources. Poultry Science, v.75, n.4, p.495-504, 1996.

EMMERT, J.L.; BAKER, D.H. Zinc stores in chickens delay the onset of zinc deficiency symptoms. Poultry Science, v.74, n.6, p.1011-1021, 1995.

FERKET, P.R.; NICHOLSON, L.; ROBERTSON, K.D. et al. Effect of level of inorganic and organic zinc and manganese on the performance and leg abnormalities of turkey toms. Poultry Science, v.71, n.1, p.60, 1992 (Abstracts) (suppl.)

FERREIRA, D.F. Sistema de análises de variância para dados balanceados (SISVAR 4. 1. - pacote computacional). Lavras: Universidade Federal de Lavras, 2000.

GEORGIEVSKII, V.I. Mineral nutrition of animals. London: Butterworts, 1982. 475p.

LEESON, S. A new look at trace mineral nutrition of poultry: can we reduce the environmental burden of poultry manure. In: ANNUAL SYMPOSIUM ON BIOTECHNOLOGY IN THE FEED INDUSTRY, 19., 2003, Nottingham. Proceedings... Nottingham: 2003. p.147-162.

LEESON, S.; SUMMERS, J.D. Nutrition of the chickens. 4.ed Guelph: University Books, 2001. p.591.

MANUAL DA LINHAGEM LOHMANN CRIA E RECRIA. Granja Planalto. Uberlândia: 2002. 6p. 
MCDOWELL, L.R. Minerals in animal and human nutrition. New York: Academic Press, 1992. 524p.

NATIONAL RESEARCH COUNCIL - NRC. Nutrient requeriment of poultry. 9.ed. Washington, D.C.: National Academic Science, 1994. $155 \mathrm{p}$.

PIMENTEL, J.L.; COOK, M.E.; GREGER, J.L. Bioavailability of zinc-methionine for chicks. Poultry Science, v.70, n.7, p.1637-1639, 1991.

ROSTAGnO, H.S.; ALBINO, L.F.T.; DONZELE, J.L. et al. Tabelas brasileiras para aves e suínos: composição de alimentos e exigências nutricionais. Viçosa, MG: Universidade Federal de Viçosa, 2000. 141p.

SCOTT, M.L.; NESHEIM, M.C.; YOUNG, R.G. Nutrition of the chicken. 3.ed. Ithaca: ML Scott and Associates, 1982. 562p.

SMITH, M.O.; SHERMAN, I.L.; MILLER, L.C. et al. Relative biological availability of manganese proteinate, manganese sulfate and manganese monoxide in broilers reared at elevated temperatures. Poultry Science, v.74, n.4, p.702-707, 1995.
UNDERWOOD, E.J. The mineral nutrition of livestock. 3.ed. Wallingford: CABI, 1999. 614p.

XAVIER, G.B.; RUTZ, F.; DIONELLO, N.J.L. et al. Performance of layers fed diets containing organic selenium, zinc and manganese, during a second cycle of production. In: ANNUAL SYMPOSIUM ON BIOTECHNOLOGY IN THE FEED INDUSTRY, 20., 2004, Lexington. Proceedings... Lexington: 2004. p. 19 . 